Science

Ben Nevis, the highest mountain in Scotland, was for many years the site of one of the most famous meteorological observatories in the world, and the discontinuance of this institution, as a result of the withdrawal of government support, was regarded by meteorologists as a serious calamity. Now it is reported that the site of the observatory is to be occupied by a tourist hotel, connected with the base of the mountain by a railway. As, even without the railway, Ben Nevis is climbed by some 15,000 people every year, it is safe to say that the commercial undertaking will be more

Fighting Mosquitoes with Bats is advocated by Dr. Charles A. R. Campbell, who claims to have exterminated countless millions of the malaria-carrying pests by means of a "bat-roost", erected alongside a large body of foul, stagnant water near San Antonio, Texas. The foost is a tall wooden structure not unlike the belfries of which bats are proverbially fond as places of residence during the day. It is provided with a tall louvered window, giving the creatures free ingress but keeping out most of the light. Dr. Campbell finds that mosquitoes are by far the commonest food of bats. Since the roost was erected, in 1911, it is claimed that both mosquitoes and chills have become scarce in the neighborhood.

Plans for the Proposed College of Tropical Agriculture in Ceylon, mention of which has already been made in these columns, have recently made rapid progress. The college is to occupy 40 acres on the left bank of the Mahaweli River, opposite the Royal Botanic Gardens at Peradeniya, at an elevation of 1,600 feet above sea-level. The main buildings, which will cost $\$ 200,000$, are to be arranged similarly to those of the Cornell College of Agriculture at Ithaca, N. Y. The lectures will provide for two groups of students; men with degrees in agriculture who wish to specialize in tropical agriculture may take a one-year course, while courses of two years or more will be given to less advanced
students.

Sleeping Sickness Investigations.-By way of a postscript to the article on sleeping sickness in the Scientific American Supplement of August 16th, it may be stated that Sir David Bruce, during a recent brief visit to England before resuming his work in Africa, declared that the sleeping sickness in Nyasaland is not the same as that of Uganda or of the West Coast, although it belongs to the same category. It is more rapid, and equally fatal, no cases of recovery having been recorded. According to the London Times, the Colonial Secretary has appointed a committee to investigate the whole subject of the spread of trypanosome infection among man and stock by tsetse flies in Africa. It is proposed to try an experiment of game destruction in a localized area, and to consider whether it is advisable to attempt the general extermination of wild animals with a view to checking these appalling diseases.

The International Institute of Agri culture now numbers 53 countries and colonies among its adherents, the latest additions to the list being Paraguay, Guatemala the Dutch East Indies, the Union of South Africa, Tripolitania, and Cyrenaica. The fourth session of th general assembly of the Institute was held in Rome cisco Guicciardini. The United States was represented cisco Guicciardini. The United States was represented
by five delegates. Probably the most important subject of debate was that concerning the promulgation of an international agreement in regard to the control of plant diseases. At present many countries, including th United States, enforce plant quarantines or otherwise contribute to the common task of checking the spread of plant diseases, but many others do not. Although of plant diseases, but many others do not. Although
the assembly passed resolutions in favor of the mainthe assembly passed resolutions in favor of the main-
tenance of government phytopathological services throughout the world, an international service seems still remote. It is hoped that the French government will soon convoke an international meeting of specialists to consider this question. To meet the growing expenses of the Institute, it was decided to increase the subscriptions of the various countries; that of the United States will hereafter be $\$ 8,000$ per annum. The recent publicawill hereafter be $\$ 8,000$ per annum. The recent publica-
tion by the Institute of the first "International Yeartion by the Institute of the first "International Year-
book of Agricultural Statisties". is said to have greatly stimulated interest in the collection of agricultural data on uniform lines, all over the world. In its work of abstracting the current literature of agriculture for its bulletins the Institute has been confronted with the difficulty of f finding equivalents in various languages fifficulty of finding equivalents in various language for many technical terms; accordingly the project is
now urder consideration of compiling a polyglot vocabulary of the principal terms employed in agriculture. The assembly passed a resolution affirming th international significance of the problem of protecting birds useful in agriculture, and suggesting its further encouragement. Other projects advocated were the
collection and dissemination of information concerning seed inspection, dry farming, and commercial fertilizers.

\section{Automobile}

Leon Bollee died recently in Paris. He was considered one of those who had done most to advance France to a front rank among the nations in the motor car industry. M. Bollee's chief claim to fame, however, is his service in 1908 to Wilbur Wright. Wright found a friend in M. Bollee who placed his services at his disposal and brought about the demonstrations at Mans which convinced the French people that heavier-than-air flying machines were an actual possibility. From then on machines were an actual possibility. F
Wright's success was assured in Europe.

Now Comes the Home Gasoline Still.-On accoun of the constantly increasing and almost prohibitive cost of gasoline as an automobile fuel, competition has become keen in the production of motors, carbureters, fuel savers and even low grade fuels. However, an English company has designed a small still by means of which an automobile owner may convert four gallon of crude oil into three and a half gallons of fuel in two hours. The oil is placed in the lower part of the still and heated by means of a burner underneath, thi burner being operated on gas or oil. The vapor arising from the heated oil is condensed in cooling coils surrounded by cold water, and is drawn off at the side into any suitable tank or vessel.

An Unusual Type of Car Heater.-To utilize some of the heat of the waste products has been the object of quite a number of persons who have sought to increase the comforts of winter motoring; but not all of them have been successful. A new type of heater that but recently has made its appearance has a number of commendable features, first and formost of which is that it may be easily attached by almost anyone who is handy with tools in a short space of time. In it simplest aspect the heater is nothing more than miniature radiator such as commonly is used for hot air, steam and hot water heating. Part of the exhaust gas is led from the exhaust pipe through the radiato whence it escapes through a relief vent to the outer air. It is in the manner of admitting the heated exhaust products to the radiator, however, that much of the novelty exists. Instead of a plain valve in the exhaust pipe there is a rotor, operated by the pressure of the exhaust gas, which in turn opens a small disk valve that admits part of the products of combustio to the radiator; thus only a small part of the gase pass into the radiator, the exact amount being governed by a small lever which in turn is connected to the disk valve so that the opening may be varied. As the heating surface is large and the tubing is fairly light only a comparatively short time need elapse before the heat is felt by the occupant of the car. To attach the devic it is merely necessary to section out a small piece of the exhaust pipe and substitute for it the combined rotor and valve that go with the heater. In the construction, two heaters are used, one in the front and one in the rear of the car, though only one need be used and it can be placed wherever the fancy of the owner and it cates.

A Semi-solid Pneumatic Tire.-A new tire has made its appearance, which is a sort of combination of solid and pneumatic. It is not unlike the ordinary tire for which it can be, substituted without alteration in existing standard tire rims. Its principal point of difference lies in its enormously thickened tread and in the unusual manner in which the tread is formed. The tread itself is four or five times as thick as the normal tread and formed in it there are a number of very deep indentations alternating on either side and overlapping slightly at the center of the tread. Between these indentations, at the sides there are other scooped out places, the purpose of which is to assist in the radiation of natural heat. By means of the deep indentations it is pointed out that the principal drawback to the solid tire, barring its lack of resiliency, of course, is eliminated. This drawback it may be explained is due to the fact that the weight of the car and its progression force a ridge, or wave, of rubber around the tire just in front of the point of contact between the tire and the road, the net result being that the tread soon loosens itself from the base and comes away. The indentations, it is claimed, do away with the travel of this wave by permitting distortion to take place and allowing for the return of the rubber to its natural form. Incidentally, their edges provide a means for the tire to bite its way through mud or water and scrape an acquaintance with the y the tire is practically non-skidding. The thickness of the tread obviously accounts for the fact that the tire is more than ordinarily resistant to punctures and blow-outs and so confident are the makers that the tire will not only stand up in service but will stand up an unusually long time to boot that they unreservedly guarantee it for 5,000 miles, 1,500 more than the manufacturer of ordinary tires. Despite the heavier tread of the tire, which is used with the ordinarr inner tube, its weight averages only about 10 per cent more than the weight of the only about 10
orthodox tire.

\title{
Centrifugal Force on Automobile Tires
}

By C. E. Palmer

A first glance it would seem that each and every A element going to make up the running gear of the modern automobile is balanced to such a nicety that any speed attainable under normal conditions would hardly be sufficient to necessitate serious consideration being given to any small, unbalanced weight. However, it has been demonstrated that even such a small weight as the tire valve is subjected to sufficient centrifugal force at high speeds, especially in racing cars, to warrant the placing of an equal weight on the opposite side of the wheel to overcome the energy developed by the rapid motion of the unbalanced valve.

The valves, including their caps, vary in weight for the different sized cars on which they are used, from $31 / 2$ to $81 / 2$ ounces, and these weights seem insignificant when compared with the weight of a wheel and rim. But when the wheel is revolved at several hundred revolutions per minute a considerable centrifugal force is exerted, acting at any point in its travel radially away from the center of the wheel. It is intended to show here the tremendous forces that are developed at different speeds if the valves are not counterbalanced by weights on the opposite sides of the wheels.

The velocity in feet per second of any particle of matter moving in a circular path with a constant radius, is represented by the product of the circumference and the number of revolutions per second. Now the centrifugal force of the same particle is the product of the weight and the square of the velocity, divided by the product of the radius and the force of gravity, which is 32.16 feet per second. Then substituting the value of $V^{2}$ in this second formula, we derive the equation, $C=$ $0.00034 W R N^{2}$ pounds, where $W$ is the weight of the body, $R$ the radius of action, and $N$ the revolutions per minute.

For the purpose of demonstrating by means of this formula, the necessity of balancing the wheels on high speed cars, values will be assumed to correspond as nearly as possible to actual practice. Let the distance from the center of the wheel to the center of gravity of the valve, be 15 inches; the weight of the valve and cap, $7 \frac{1}{2}$ ounces or 0.468 pounds; and the diameter of the tire, 36 inches. Cars which will not attain a speed of 60 miles per hour are unusual, and it is an every day occurrence for racers to maintain a speed of 75 or 80 miles per hour throughout a long contest or tria run. As a speed that is not at all unusual, let us assume 70 miles per hour for the first example. This is equivalent to 6,160 feet per minute, and in traveling at this speed a wheel with a 36-inch tire will make 654 revolutions per minute. S

$$
C=0.00034 \times 0.468 \times 1.25 \times 654 \times 654=85.1 \mathrm{lbs} .
$$

In other words a $7 \frac{1}{2}$-ounce valve on a 36 -inch wheel traveling at $\mathbf{7 0}$ miles per hour will exert a lifting force of over 85 pounds when the valve reaches the top of the wheel. In case both valves of either pair of wheels are in the same relative position with regard to the axle, they will exert a combined lifting force of $\mathbf{1 7 0 . 2}$ pounds, and if they are opposite each other there will be a seesawing force acting on the car. Considering that there are four of these valves it is easily seen that they will exert forces in various and constantly changing directions as the wheels shift their relative positions in rounding turns in the road.

Since the centrifugal force varies as the square of the speed it requires only a slight increase in the speed to make a large increase in the force exerted. For in stance, if the car travels at 75 miles per hour the force is increased to 97.5 pounds, and at 80 miles, which is frequently attained, the force on each valve will be 111.3 pounds, while at only 40 miles the force is 28 pounds. In a car going at the fastest rate of speed yet attained by man, 142 miles per hour, the force exerted by the valve is nearly 400 pounds.

Considering these almost neglected forces it is easily seen that some cognizance of them should be taken the same as is done is designing balanced wheels of stationary machinery. That racing drivers are coming to realize what these neglected forces might mean in case of an accident and loss of control of the car, is shown by the fact that a famous English driver has equipped each of his tires with two valves instead of one, the valves being placed on opposite sides of the tires in order to provide a perfect balance. It is said that the car will run at 80 miles per hour and hold the road remarkably well, where before it skidded badly at high speeds and was very difficult to control. Many American drivers also balance their valves by placing meta weights on the rim or felloes of the wheels opposite the valves.

Elk in Utah.-Elk have been found in the Uinta national forest, Utah, for the first time in many years. Since they are not from shipments from the Jackson 


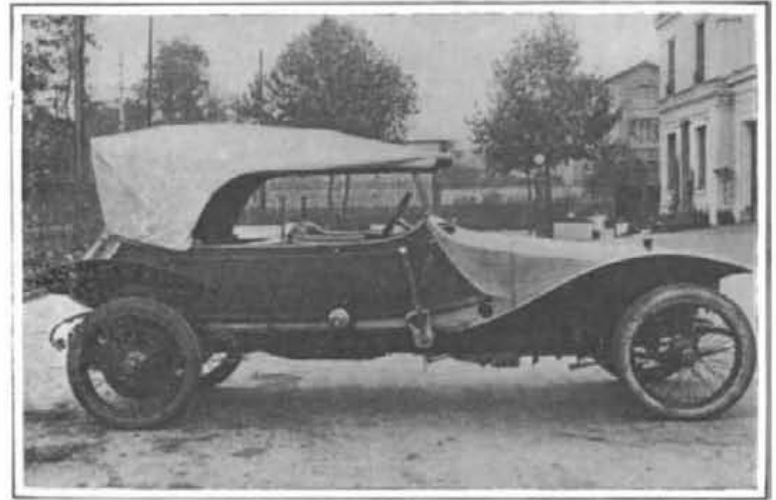

An interesting example of the "boat type" body. The peculiar feature of the design lies in its ra:n-like for-
ward lines and its torpedo stern; it is styled a skiff-torpedo Lightness of construction evidently was sought, for running
boardg are missing and the mudguards are small.

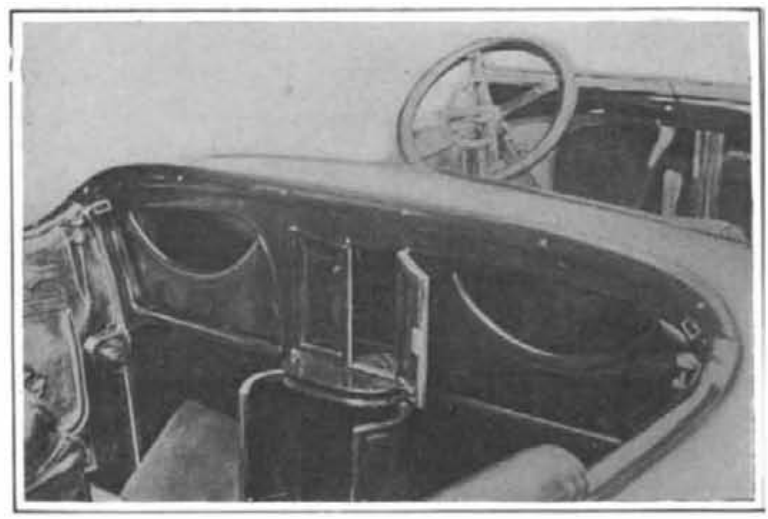

Ingenious storage spaces that satisfy a real demand. To provide for the safe carrying of goggles, gloves and the hundred and one other motoring necessities is not always
easy, though the designer of this body has not found it par-
ticularly difficult. In the average American car, storage

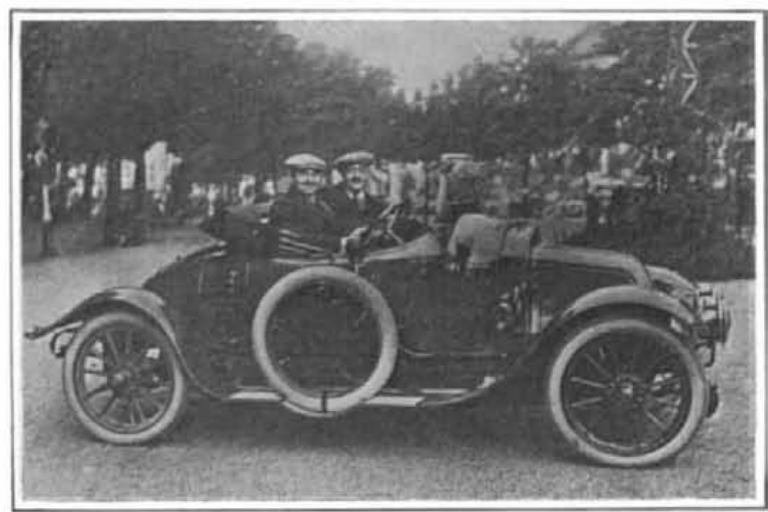

Side view of lately developed runabout model. Compactness is the predominating feature of this vehicle
which somewhat resembles a great black beetle, what with its sloping front and rear and its low-bung chassis. The is unusual.

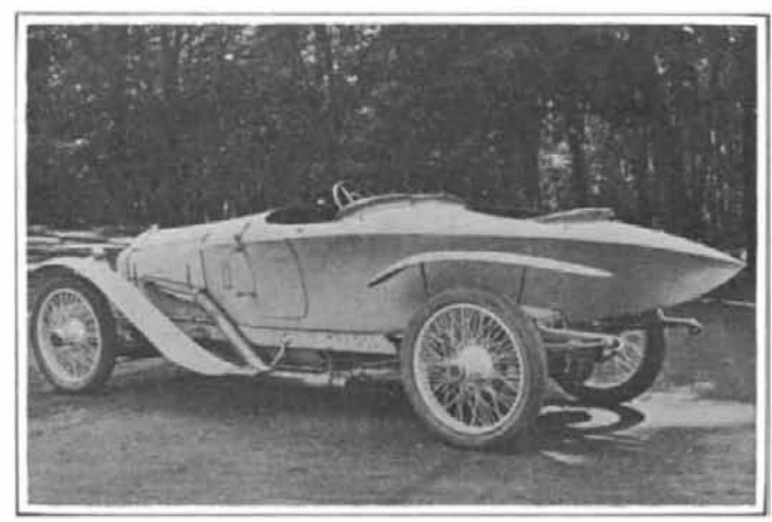

Another of the curious foreign "boat" bodies. The exaggerated flare to the top-sides, the tiny after cockpit
and the peculiar stern all proclaim the marine origin of the type. Mudguards are reduced to the minimum, it will be noted, and there are no side entrances to the single rea
seat; running lamps are mounted atop the head lamps.

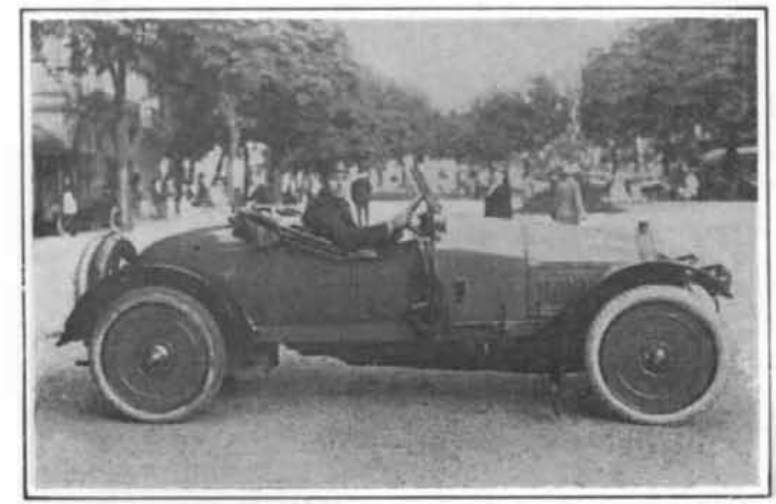

A representative type of fast foreign runabout. Disked wheels, practically unknown in America, serve to
reduce wind resistance and to promote cleanliness; they are cairly common abroad where little attention is paid to con rear effect and the lowness of the chassis.

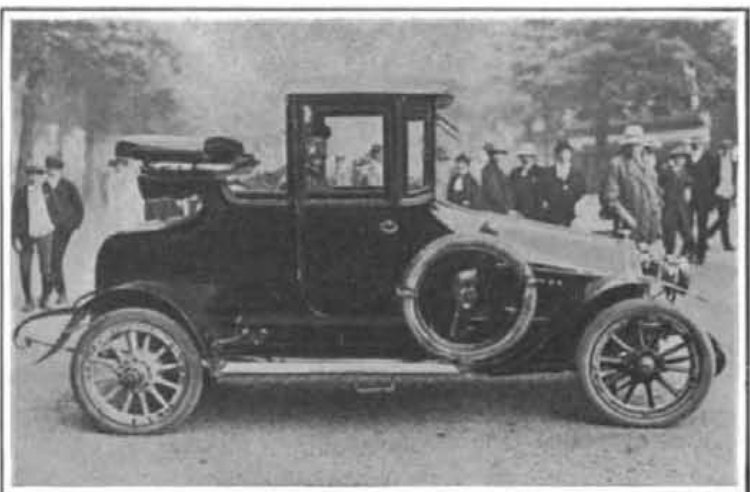

Modified type of two-passenger coupé model. When closed, this body has much the appearance of a "con-
ning tower." It is a good example of the European practice of sloping the engine hood sharply to blend with the lines of

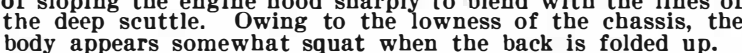

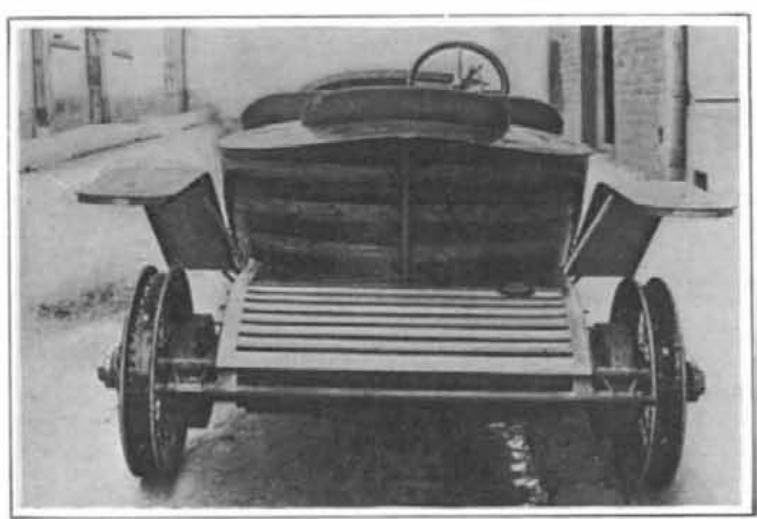

Is closer resemblance to a real. boat possible? The fidelity with which the boat builder's art bas been imitated in this body is truly astonishing;
erly built up of a number of "skins;" decks are laid in true
nautical fashion and even the cockpit has a coanling. Note the protected fuel tank.

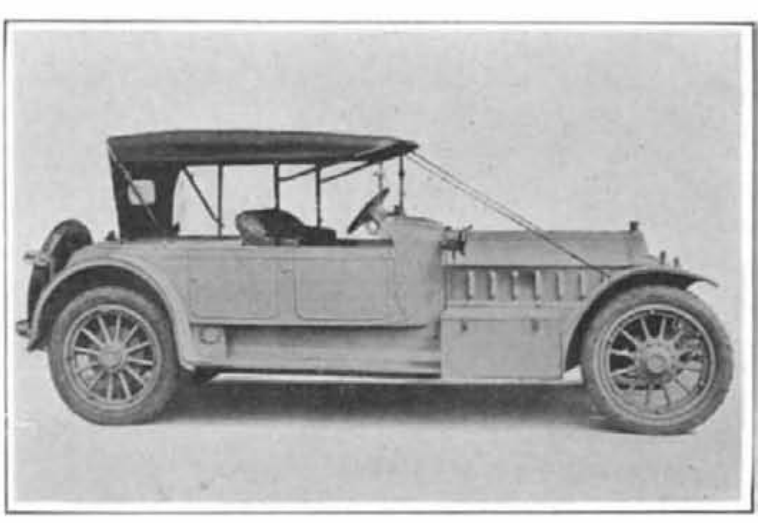

American three-passenger car that is different. The third seat at the rear closes up out of sight and when
closed is wateroroof besides it there are commodious lockers. 'The position of the tool box is worthy of note, fo though
board.

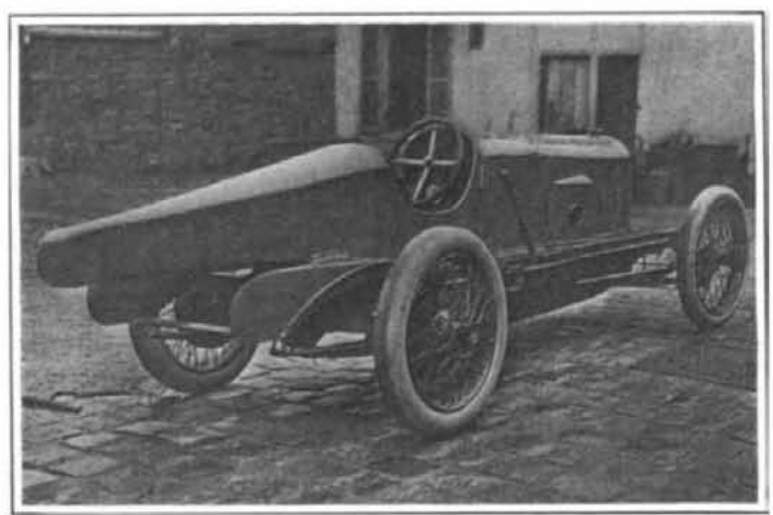

How foreign designers reduce wind resistance. Type of racing body in which the stream-line principle is well exemplifed, the theory being that the pressure at the
rear nearly neutralizes the head resistance: The extreme to which the idea is carried is indicated hy the rudder-

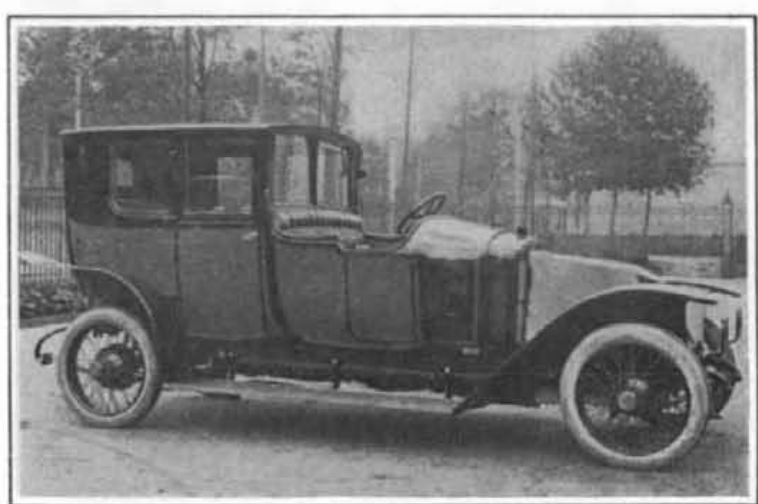

The exaggerated coupé type of town vehicle.

The apparent great length of the chassis is accounted fo
by the fact that more than half the space is taken up by engine and driver. The abrupt. unfinished appearance of
the inclosed part off sets the elaborate attempt at line blend
ing at the front.

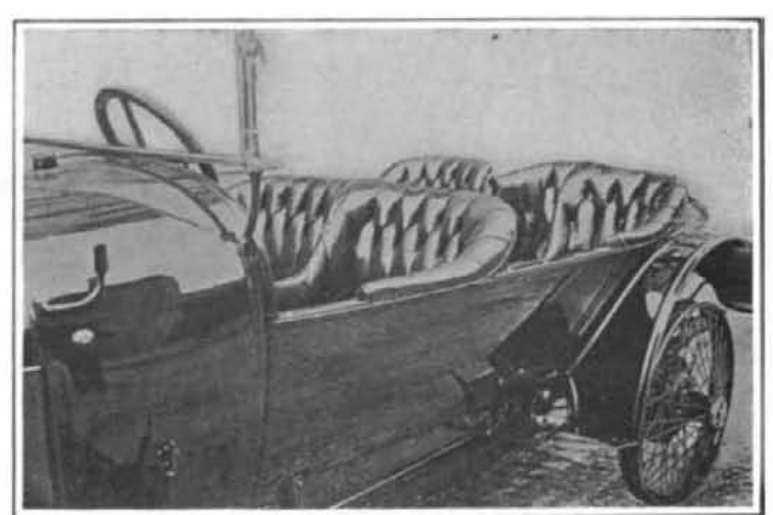

A clever solution of the seating problem. In this body, the designer has placed the tonneau seats en
echelon by way of giving all three occupaints plenty of elbow room. The design, incidentally provides for sociability
among the passengers; conversation is facilitated; for no
one is required to lean over any one else.

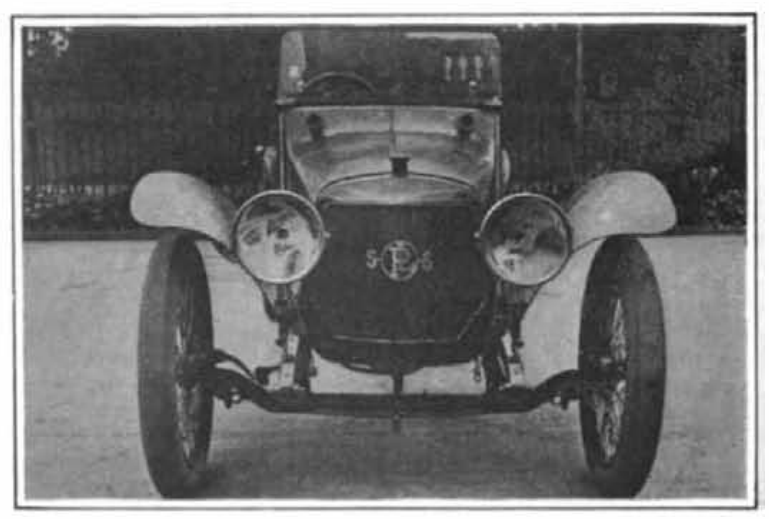

Dodging the conventional in front elevations. The resenblance to an aeroplane with its widespread wings in unmistakable. The miniature "side" lamps, set into the
dash, are in marked contrast to the powerful head lamps. The windshield, unbound at the top, reflects a well-defined
tendency in design. The body is styled a skiff-torpedo.

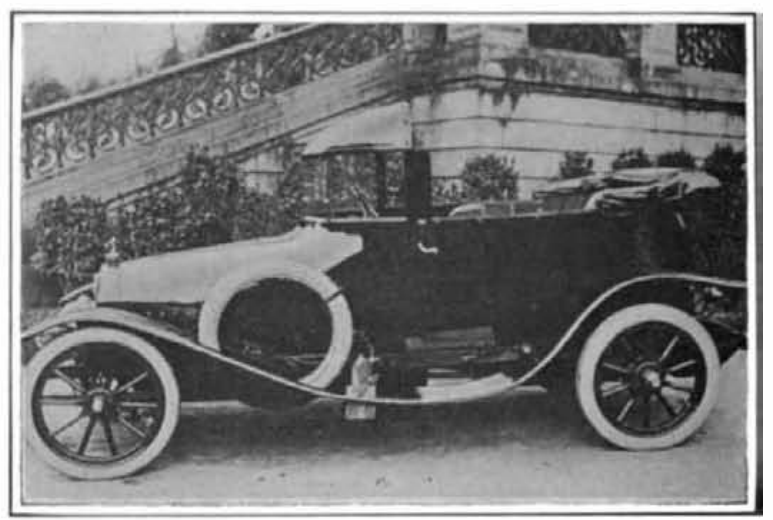

Clever combination of sedan and limousine. When the top is folded back, as shown, the body is to all raised the occupants may be as snug as the proverbial bug. severity of American practice !

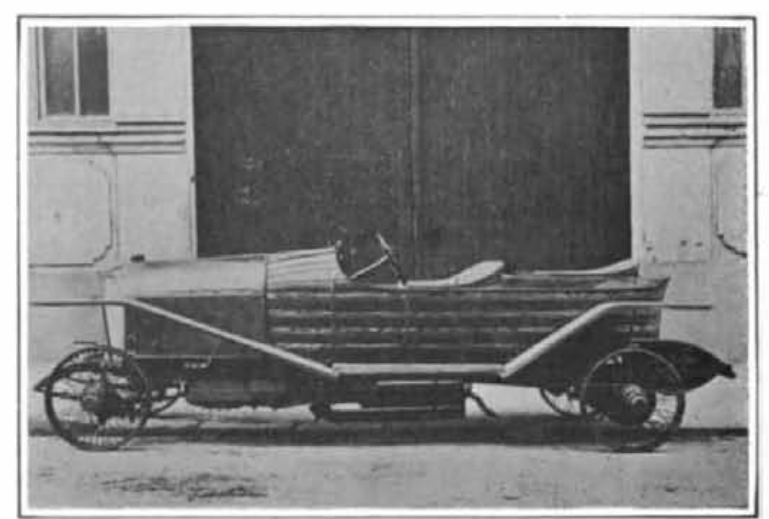

Another view showing lines of the "terra marine" body. From the side, the resemblance to a boat is even more pronounced; the lines are those of the typical, fast runabout as to obscure the engine hood. This body caused a genuine

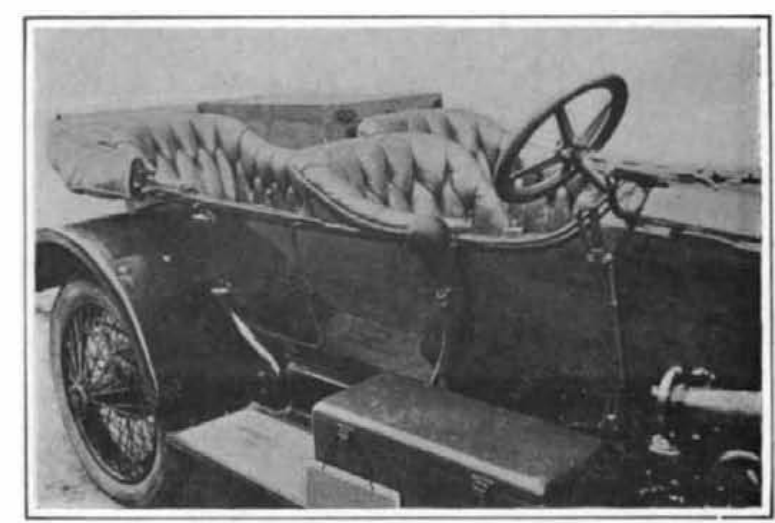

Brand new arrangement of seats and storage space. of room, and balf the tonneau is reserved for luggage. The body is a utilitarian one, though the passenger capacity 